\title{
Matrix Order Indices in Statistical Mechanics
}

\author{
V.I. Yukalov \\ Research Center for Optics and Photonics \\ Instituto de Fisica de São Carlos, Universidade de São Paulo \\ Caixa Postal 369, São Carlos, São Paulo 13560-970, Brazil \\ and \\ Bogolubov Laboratory of Theoretical Physics \\ Joint Institute for Nuclear Research, Dubna 141980, Russia
}

\begin{abstract}
A new notion is introduced of matrix order indices which relate the matrix norm and its trace. These indices can be defined for any given matrix. They are especially important for matrices describing many-body systems, equilibrium as well as nonequilibrium, for which the indices present a quantitative measure of the level of ordering. They characterize not only the long-range order, but also midrange order. In the latter case, when order parameters do not exist, the matrix indices are well defined, providing an explicit classification of various mid-range orders. The matrix order indices are suitable for describing phase transitions with both off-diagonal and diagonal order. Contrary to order parameters whose correct definition requires the thermodynamic limit, the matrix indices do not necessarily need the latter. Because of this, such indices can distinguish between different phases of finite systems, thus, allowing for the classification of crossover phase transitions.
\end{abstract}

PACS: 02.10.Yn; 05.70.Fh; 64.60.Cn

Keywords: Statistical systems; Phase transitions; Classification of orders 


\section{Introduction}

Matrices are common objects in quantum physics, where all observable quantities are related with matrix representations of operators. In statistical mechanics, a special role is played by reduced density matrices [1-3]. The eigenvalues of the latter are known to be associated with a possible existence of long-range order called off-diagonal long-range order [4-6]. This linkage between the eigenvalues and the order has been mathematically formalized by means of order indices for reduced density matrices [79]. These indices turned out to be a convenient tool for characterizing the arising order related to Bose-Einstein condensation and superconductivity [3]. There are, however, several limitations not permitting one to employ these indices for describing other types of order. Among the most important problems, the following are to be solved: (i) How the appearance of magnetic order could be noticed in the behaviour of the indices? (ii) How to describe phase transitions associated with diagonal order, such as crystallization? (iii) How to characterize crossover-type phase transitions occurring in finite systems?

In the present paper a new notion of matrix order indices is introduced. This notion is an essential generalization of the order indices for reduced density matrices [7-9]. Contrary to the latter, the matrix indices are defined for any given matrix of arbitrary nature. Because of this generality, the matrix indices are suitable for characterizing any types of phase transitions, accompanied by any kinds of order, diagonal as well as off-diagonal, long-range as well as mid-range, in infinite as well as in finite systems. In section 2, a general mathematical definition of the matrix order indices is given. These are the quantities linking the norm and the trace of a given matrix whose nature can be arbitrary. The possibility of using different norms is discussed. In section 3 , the matrix indices of self-adjoint semipositive matrices are considered, because of the special role played by such matrices in physical applications. The convenience of employing the introduced matrix indices for quantitatively comparing various kinds of mid-range orders is illustrated in section 4. In section 5, Bose-Einstein condensation in confined systems is analysed, which is important for describing the condensation of trapped atoms. Section 6 demonstrates how the matrix indices must be defined in order to treat magnetic phase transitions. Finally, in section 7, it is shown how the phase transitions, such as crystallization, characterized by diagonal order, can be described by an appropriate definition of the matrix order indices.

\section{Matrix order indices}

Let us have a matrix $\hat{M}$ of arbitrary nature, with a norm $\|\hat{M}\|$ and trace $\operatorname{Tr} \hat{M}$. The matrix order index is defined as

$$
\omega_{M} \equiv \frac{\ln || \hat{M} \|}{\ln |\operatorname{Tr} \hat{M}|}
$$


We shall say that among two given matrices that one is better ordered whose order index is larger. As follows from definition (1), for norm-one and traceless matrices, one has $\omega_{M}=0$.

The value of the matrix index (1) depends on the type of the norm $\|\hat{M}\|$ used. Assume that a matrix $\hat{M}$ acts on a normed space $\{\varphi\}$ of functions having a vector norm $|\varphi|$. The matrix norm, associated with a vector norm $|\varphi|$, is given by

$$
\|\hat{M}\| \equiv \sup _{\varphi} \frac{|\hat{M} \varphi|}{|\varphi|} .
$$

Here and in what follows, it is implied that $|\varphi| \neq 0$. If $\{\varphi\}$ is a Hilbert space, with a scalar product $(\varphi, \varphi)$, the latter naturally generates the Hermitian vector norm $|\varphi|=$ $\sqrt{(\varphi, \varphi)}$. Then, for a matrix $\hat{M}$, the Hermitian norm is

$$
\|\hat{M}\| \equiv \sup _{\varphi} \frac{\left(\varphi, \hat{M}^{+} \hat{M} \varphi\right)^{1 / 2}}{|\varphi|}
$$

where $\hat{M}^{+}$is a Hermitian conjugated matrix. Supposing that the matrix $\hat{M}$ possesses a spectrm $\left\{\mu_{n}\right\}$ of eigenvalues, one has the spectral norm

$$
\| \hat{M}||=\sup _{n}\left|\mu_{n}\right| .
$$

More generally, the spectral norm is defined as the square root of the largest eigenvalue of the matrix $\hat{M}^{+} M$. When $\hat{M}=\hat{M}^{+}$is a self-adjoint and semipositive matrix, then its eigenvalues are semipositive, $\mu_{n} \geq 0$. In that case, the spectral norm becomes

$$
\|\hat{M}\|=\sup _{\varphi} \frac{(\varphi, \hat{M} \varphi)}{|\varphi|}=\sup _{n} \mu_{n} .
$$

Among other matrix norms, for physical applications one may employ the trace norm

$$
\|\hat{M}\|^{\prime} \equiv\left(\operatorname{Tr} \hat{M}^{+} \hat{M}\right)^{1 / 2}
$$

which is also called the Euclidean norm. Definition (5) may be convenient for calculations, since the trace does not depend on a matrix representation. With a spectrum $\left\{\mu_{n}\right\}$ of $\hat{M}$, the trace norm (5) reads

$$
\|\hat{M}\|^{\prime}=\left(\sum_{n}\left|\mu_{n}\right|^{2}\right)^{1 / 2}
$$

As follows from the above definitions, $\|\hat{M}\| \leq\|\hat{M}\|^{\prime}$, hence one has to distinguish between the associated indices $\omega_{M}$ and $\omega_{M}^{\prime}$, for which $\omega_{M} \leq \omega_{M}^{\prime}$. 


\section{Self-adjoint semipositive matrices}

Self-adjoint matrices play a special role in physical applications, because of which they need to be studied more attentively. Let $\mathcal{X}=\{x\}$ be an arithmetic space of physical coordinates and let $t \in[0, \infty)$ denote time. The set $x$ may include Cartesian coordinates, spin, isospin, and other quantum variables. Assume that an algebra of local observables $\mathcal{A}=\{A(x, t)\}$ and an algebra of field operators $\Psi=\left\{\psi(x, t), \psi^{\dagger}(x, t)\right\}$ are given on a Fock space $\mathcal{F}$. The direct sum $\tilde{\mathcal{A}} \equiv \mathcal{A} \oplus \Psi$ denotes an extended local algebra.

Consider a system of $p$ objects that, for concreteness, will be called particles. The set $x^{p} \equiv\left\{x_{1}, x_{2}, \ldots, x_{p}\right\}$ of particle coordinates pertains to the space $\mathcal{X}^{p} \equiv \mathcal{X}_{1} \times \mathcal{X}_{2} \times$ $\ldots \times \mathcal{X}_{p}$, which is a $p$-fold tenzor product. In a Hilbert space of complex functions $\varphi_{p}\left(x^{p}\right)$, the scalar product is given by the integral

$$
\left(\varphi_{p}, \varphi_{p}^{\prime}\right) \equiv \int \varphi_{p}^{*}\left(x^{p}\right) \varphi_{p}^{\prime}\left(x^{p}\right) d x^{p}
$$

where $d x^{p} \equiv d x_{1} d x_{2} \ldots d x_{p}$. In general, $p=1,2, \ldots$

Introduce the function

$$
D_{p}\left(A, x^{p}, \bar{x}^{p}, t\right) \equiv \operatorname{Tr}_{\mathcal{F}} A\left(x_{1}\right) \ldots A\left(x_{p}\right) \hat{\rho}(t) A^{+}\left(\bar{x}_{p}\right) \ldots A^{+}\left(\bar{x}_{1}\right),
$$

where the trace is taken over the Fock space $\mathcal{F}, A(x) \equiv A(x, 0)$ is a member of the extended local algebra $\tilde{\mathcal{A}}$, and $\hat{\rho}(t)$ is a statistical operator. It is admissible to treat the function (6) as an element of the matrix

$$
\hat{D}_{p}(A, t) \equiv\left[D_{p}\left(A, x^{p}, \bar{x}^{p}, t\right)\right]
$$

with respect to the variables $x^{p}$. By construction (6), the matrix (7) is self-adjoint and positive semidefinite. The action of the matrix (7) on a column $\varphi_{p} \equiv\left[\varphi_{p}\left(x^{p}\right)\right]$ gives the column

$$
\hat{D}_{p}(A, t) \varphi_{p}=\left[\int D_{p}\left(A, x^{p}, \bar{x}^{p}, t\right) \varphi_{p}\left(\bar{x}^{p}\right) d \bar{x}^{p}\right] .
$$

A linear envelope of the vectors $\varphi_{p}$ forms a Hilbert space $\mathcal{H}_{p}=\mathcal{L}\left\{\varphi_{p}\right\}$ with a scalar product $\varphi_{p}^{+} \varphi_{p}^{\prime} \equiv\left(\varphi_{p}, \varphi_{p}^{\prime}\right)$. Each matrix $(7)$ acts on $\mathcal{H}_{p}$. The spectral norm for a self-adjoint semipositive matrix can be written as

$$
\left\|\hat{D}_{p}(A, t)\right\| \equiv \sup _{\varphi_{p}} \frac{\varphi_{p}^{+} \hat{D}_{p}(A, t) \varphi_{p}}{\varphi_{p}^{+} \varphi_{p}} .
$$

The trace over the variables $x^{p}$ is

$$
\operatorname{Tr} \hat{D}_{p}(A, t) \equiv \int D_{p}\left(A, x^{p}, x^{p}, t\right) d x^{p} .
$$

According to Eq. (1), one may introduce the matrix indices

$$
\omega_{p}(A, t) \equiv \frac{\ln || \hat{D}_{p}(A, t)||}{\ln \left|\operatorname{Tr} \hat{D}_{p}(A, t)\right|} .
$$


For a semipositive matrix, one has

$$
\left\|\hat{D}_{p}(A, t)\right\| \leq \operatorname{Tr} \hat{D}_{p}(A, t),
$$

because of which

$$
\omega_{p}(A, t) \leq 1
$$

Similarly, employing the trace norm (5), one obtains the matrix indices $\omega_{p}^{\prime}(A, t)$ for which inequality (11) is also valid.

For constructing a matrix (7), one may choose any representative of the extended local algebra $\tilde{\mathcal{A}}$. In particular, opting for the field operators $\psi(x, t)$, we come to the reduced density matrices

$$
\hat{\rho}_{p}(t) \equiv\left[\rho_{p}\left(x^{p}, \bar{x}^{p}, t\right)\right]
$$

with the elements

$$
\rho_{p}\left(x^{p}, \bar{x}^{p}, t\right) \equiv D_{p}\left(\psi, x^{p}, \bar{x}^{p}, t\right)=\operatorname{Tr}_{\mathcal{F}} \psi\left(x_{1}\right) \ldots \psi\left(x_{p}\right) \hat{\rho}(t) \psi^{\dagger}\left(\bar{x}_{p}\right) \ldots \psi^{\dagger}\left(\bar{x}_{1}\right) .
$$

The spectral norm for the $p$-particle density matrix (12) is

$$
\left\|\hat{\rho}_{p}(t)\right\|=\sup _{n} \rho_{p n}(t)
$$

with $\left\{\rho_{p n}(t)\right\}$ being the related spectrum.

The number of particles, $N$, in a statistical system is assumed to be large. Then, in the trace

$$
\operatorname{Tr} \hat{\rho}_{p}(t)=\frac{N !}{(N-p) !} \quad(p=1,2, \ldots, N)
$$

one may use the Stirling formula $N ! \simeq \sqrt{2 \pi N} N^{N} e^{-N}$, which, under $p$ fixed and $N \gg 1$, yields

$$
\frac{N !}{(N-p) !} \simeq \frac{N^{p}}{e^{p}} .
$$

Hence, the matrix index (10) becomes

$$
\omega_{p}(\psi, t) \simeq \frac{\ln \left\|\hat{\rho}_{p}(t)\right\|}{p \ln N}
$$

for $N \gg 1$.

For equilibrium statistical systems, one often considers the thermodynamic limit

$$
N \rightarrow \infty, \quad V \rightarrow \infty, \quad \frac{N}{V} \rightarrow \rho,
$$

in which $N$ is the number of particles in the volume $V$ and the limiting density $\rho$ is finite, $0<\rho<\infty$. In equilibrium, the matrix index (15) does not depend on time, becoming

$$
\omega_{p}(\psi) \simeq \frac{\ln \left\|\hat{\rho}_{p}\right\|}{p \ln N}
$$


as $N \gg 1$. Note that for calculating the index (17) it is not compulsory to take the actual thermodynamic limit (16) by setting $N \rightarrow \infty$, but Eq. (17) can be used for finite systems, provided that $N \gg 1$.

From the properties of the reduced density matrices [3], we know that for bosons $\left\|\hat{\rho}_{p}\right\| \leq N^{p}$. const, hence $\omega_{p}(\psi) \leq 1$, in agreement with the general inequality (11). The equality

$$
\left.\omega_{p}(\psi)=1 \quad \text { (boson long }- \text { range order }\right)
$$

corresponds to long-range order in a bosonic system, as that occurring at Bose-Einstein condensation of dilute gases.

Reduced density matrices for fermionic systems [3] satisfy the inequalities

$$
\left\|\hat{\rho}_{2 p}\right\| \leq N^{p} \cdot \text { const }, \quad\left\|\hat{\rho}_{2 p-1}\right\| \leq N^{p-1} \cdot \text { const },
$$

because of which for the matrix indices we have

$$
\omega_{2 p}(\psi) \leq \frac{1}{2}, \quad \omega_{2 p-1}(\psi) \leq \frac{p-1}{2 p-1} .
$$

The limiting case

$$
\omega_{2 p}(\psi)=\frac{1}{2} \quad(\text { even long }- \text { range order })
$$

happens when even long-range order develops in a fermionic system, like that arising in superconductors. When Eq. (20) is valid, then

$$
\omega_{2 p-1}(\psi)=\frac{p-1}{2 p-1} \quad(p=1,2, \ldots, N) .
$$

For large $p$, this tends to the value (20), since

$$
\omega_{2 p-1}(\psi) \simeq \frac{1}{2}-\frac{1}{4 p} \quad(p \gg 1) .
$$

Even long-range order may also happen for bosonic systems.

The matrix indices, introduced above, are different from the indices for reduced density matrices, defined in the earlier papers [7-9]. The main difference is that, in the case of long-range order, the matrix indices $\omega_{p}(\psi)$ or $\omega_{2 p}(\psi)$ do not depend on $p$, displaying a kind of resonance, as follows from Eqs. (18) and (20).

\section{Finite momentum condensation}

In order to explicitly illustrate the calculation of the matrix order indices, for both long-range and mid-range orders, let us consider a Bose system with finite momentum

condensate [10-12]. Such a condensate consists of particles having a finite modulus of 
momentum $k_{0}=\left|\mathbf{k}_{0}\right|$, with random directions of the vector $\mathbf{k}_{0}$. The related condensate density matrix reads

$$
\rho_{0}\left(\mathbf{r}, \mathbf{r}^{\prime}\right)=\frac{\rho_{0}}{\int d \Omega\left(\mathbf{k}_{0}\right)} \int e^{i \mathbf{k}_{0} \cdot\left(\mathbf{r}-\mathbf{r}^{\prime}\right)} d \Omega\left(\mathbf{k}_{0}\right)
$$

where $\rho_{0} \equiv N_{0} / V$ is the condensate density and $\Omega\left(\mathbf{k}_{0}\right)$ is a spherical angle associated with $\mathbf{k}_{0}$. The single-particle density matrix tends, as the distance between the points $\mathbf{r}$ and $\mathbf{r}^{\prime}$ increases, to the condensate matrix (21),

$$
\rho_{1}\left(\mathbf{r}, \mathbf{r}^{\prime}\right) \simeq \rho_{0}\left(\mathbf{r}, \mathbf{r}^{\prime}\right) \quad\left(\left|\mathbf{r}-\mathbf{r}^{\prime}\right| \rightarrow \infty\right)
$$

In a particuar case of zero momentum $\mathbf{k}_{0}=0$, Eq. (21) reduces to the constant density

$$
\rho_{0}\left(\mathbf{r}, \mathbf{r}^{\prime}\right)=\rho_{0} \quad\left(\mathbf{k}_{0} \equiv 0\right)
$$

corresponding to the usual Bose-Einstein condensate with the order index (18). But for the finite-momentum condensate, the situation is more elaborate and depends on the space dimensionality.

\subsection{Three-dimensional case}

In the three-dimensional space, the condensate matrix (21) reads

$$
\rho_{0}\left(\mathbf{r}, \mathbf{r}^{\prime}\right)=\rho_{0} \frac{\sin k_{0}\left|\mathbf{r}-\mathbf{r}^{\prime}\right|}{k_{0}\left|\mathbf{r}-\mathbf{r}^{\prime}\right|}
$$

For the considered uniform system, the natural single-particle states, which are the eigenfunctions of the single-particle density matrix, are the plane waves

$$
\varphi_{k}(\mathbf{r})=\frac{1}{\sqrt{V}} e^{i \mathbf{k} \cdot \mathbf{r}}
$$

Introducing the notation for the radius of the system,

$$
R \equiv\left(\frac{3 N}{4 \pi \rho}\right)^{1 / 3}
$$

for the matrix elements of $\hat{\rho}_{0}=\left[\rho_{0}\left(\mathbf{r}, \mathbf{r}^{\prime}\right)\right]$ we have

$$
\left(\varphi_{k}, \hat{\rho}_{0} \varphi_{k}\right)=2 \pi \frac{\rho_{0} R}{k k_{0}}\left[\frac{\sin \left(k-k_{0}\right) R}{\left(k-k_{0}\right) R}-\frac{\sin \left(k+k_{0}\right) R}{\left(k+k_{0}\right) R}\right] .
$$

In particular, if $k_{0} \rightarrow 0$, then

$$
\lim _{k_{0} \rightarrow 0}\left(\varphi_{k}, \hat{\rho}_{0} \varphi_{k}\right)=4 \pi \frac{\rho_{0} R}{k^{2}}\left(\frac{\sin k R}{k R}-\cos k R\right) .
$$


From here, the largest eigenvalue of the single-particle density matrix is

$$
\sup _{k} \lim _{k_{0} \rightarrow 0}\left(\varphi_{k}, \hat{\rho}_{0} \varphi_{k}\right)=N_{0}
$$

which results in the order index (18).

However, for $k_{0} \neq 0$, we find

$$
\sup _{k}\left(\varphi_{k}, \hat{\rho}_{0} \varphi_{k}\right)=2 \pi \frac{\rho_{0} R}{k_{0}^{2}}\left[1-\frac{\sin \left(2 k_{0} R\right)}{2 k_{0} R}\right] .
$$

For large systems, such that $k_{0} R \gg 1$, we obtain

$$
\left\|\hat{\rho}_{1}\right\| \simeq 2 \pi \frac{\rho_{0}}{k_{0}^{2}}\left(\frac{3 N}{4 \pi \rho}\right)^{1 / 3}
$$

which requires that the number of particles be large,

$$
N \gg \frac{4 \pi \rho}{3 k_{0}^{3}} .
$$

Thus, for the order index of the single-particle matrix, we obtain $\omega_{1}(\psi)=1 / 3$. In the general case, exploiting the relation

$$
\left\|\hat{\rho}_{p}\right\| \sim\left\|\hat{\rho}_{1}\right\|^{p},
$$

valid for asymptotically large $N$, we have the order indices (17) as

$$
\omega_{p}(\psi)=\frac{1}{3} .
$$

This value of the order indices corresponds to mid-range order.

If, instead of the spectral norm (4), one uses the trace norm (5), then the order indices for reduced density matrices are different. To calculate such indices, we notice that

$$
\operatorname{Tr} \hat{\rho}_{0}^{2}=2 \pi V \frac{\rho_{0}^{2} R}{k_{0}^{2}}\left[1-\frac{\sin \left(2 k_{0} R\right)}{2 k_{0} R}\right] .
$$

For the standard condensate with $k_{0}=0$, we have $\operatorname{Tr} \hat{\rho}_{0}^{2}=N_{0}^{2}$, from where $\omega_{1}^{\prime}(\psi)=1$, which coincides with $\omega_{1}(\psi)=1$. But if $k_{0} \neq 0$ and the system is large, such that $k_{0} R \gg 1$, then we find

$$
\left\|\hat{\rho}_{1}\right\|^{\prime} \simeq \sqrt{\frac{8}{3}} \pi \frac{\rho_{0}}{k_{0}}\left(\frac{3 N}{4 \pi \rho}\right)^{2 / 3} .
$$

Finally, we obtain

$$
\omega_{p}^{\prime}(\psi)=\frac{2}{3},
$$

which differs from the index (25). 


\subsection{Two-dimensional case}

The condensate density matrix $(21)$ is

$$
\rho_{0}\left(\mathbf{r}, \mathbf{r}^{\prime}\right)=\rho_{0} J_{0}\left(k_{0}\left|\mathbf{r}-\mathbf{r}^{\prime}\right|\right),
$$

where

$$
J_{n}(z) \equiv \frac{(-1)^{n}}{\pi} \int_{0}^{\pi} e^{i z \cos \varphi} \cos (n \varphi) d \varphi
$$

is the first-type Bessel function, with $n=0,1,2, \ldots$ Using the relation

$$
J_{n}\left(z e^{i \pi m}\right)=e^{i \pi m n} J_{n}(z)
$$

in which $m=0, \pm 1, \pm 2, \ldots$, and the integral

$$
\int x J_{n}(a x) J_{n}(b x) d x=\frac{x}{a^{2}-b^{2}}\left[a J_{n+1}(a x) J_{n}(b x)-b J_{n}(a x) J_{n+1}(b x)\right],
$$

we find

$$
\left(\varphi_{k}, \hat{\rho}_{0} \varphi_{k}\right)=\frac{2 \pi \rho_{0} R}{k^{2}-k_{0}^{2}}\left[k J_{1}(k R) J_{0}\left(k_{0} R\right)-k_{0} J_{0}(k R) J_{1}\left(k_{0} R\right)\right]
$$

where

$$
R \equiv\left(\frac{N}{\pi \rho}\right)^{1 / 2}
$$

is the system radius.

When $k_{0} \rightarrow 0$, then

$$
\lim _{k_{0} \rightarrow 0}\left(\varphi_{k}, \hat{\rho}_{0} \varphi_{k}\right)=2 \pi \frac{\rho_{0} R}{k} J_{1}(k R)
$$

Because of the asymptotic equation

$$
J_{n}(z) \simeq \frac{1}{n !}\left(\frac{z}{2}\right)^{n} \quad(z \rightarrow 0)
$$

it follows that

$$
\sup _{k} 2 \pi \frac{\rho_{0} R}{k} J_{1}(k R)=\pi R^{2} \rho_{0}=N_{0} .
$$

And we again return to the indices (18).

However, for the finite-momentum condensate, with $k_{0} \neq 0$, we have

$$
\sup _{k}\left(\varphi_{k}, \hat{\rho}_{0} \varphi_{k}\right)=\pi \rho_{0} R^{2}\left[J_{0}^{2}\left(k_{0} R\right)-\frac{J_{0}\left(k_{0} R\right) J_{1}\left(k_{0} R\right)}{k_{0} R}+J_{1}^{2}\left(k_{0} R\right)\right],
$$

where the equations

$$
z \frac{d J_{n}(z)}{d z}=n J_{n}(z)-z J_{n+1}(z)=z J_{n-1}(z)-n J_{n}(z)
$$


have been used. For a large system, such that $k_{0} R \gg 1$, we invoke the asymptotic form

$$
J_{n}(z) \simeq \sqrt{\frac{2}{\pi z}} \cos \left(z-\frac{\pi}{2} n-\frac{\pi}{4}\right) \quad(z \rightarrow \infty) .
$$

Then, for a system of many particles, with

$$
N \gg \frac{\pi \rho}{k_{0}^{2}},
$$

we get

$$
\left\|\hat{\rho}_{1}\right\| \simeq 4 \frac{\rho_{0}}{k_{0}}\left(\frac{N}{\pi \rho}\right)^{1 / 2} \cos ^{2}\left(k_{0} R\right) .
$$

For the order indices we obtain

$$
\omega_{p}(\psi)=\frac{1}{2} .
$$

If calculating the order indices, one opts for the trace norm (5), then the resulting $\omega_{p}^{\prime}(\psi)$ differs from the indices $(29)$. Thus, using the relations

$$
\begin{gathered}
J_{-n}(z)=(-1)^{n} J_{n}(z) \\
\int x J_{n}^{2}(a x) d x=\frac{x^{2}}{2}\left[J_{n}^{2}(a x)-J_{n-1}(a x) J_{n+1}(a x)\right],
\end{gathered}
$$

we have

$$
\operatorname{Tr} \hat{\rho}_{0}^{2}=\left(\rho_{0} \pi R^{2}\right)\left[J_{0}^{2}\left(k_{0} R\right)+J_{1}^{2}\left(k_{0} R\right)\right] .
$$

The indices $\omega_{p}^{\prime}(\psi)$ coincide with $\omega_{p}(\psi)=1$ only if $k_{0}=0$, since then $\operatorname{Tr} \hat{\rho}_{0}^{2}=N_{0}^{2}$. But for $k_{0} \neq 0$, again keeping in mind a large system, with $k_{0} R \gg 1$, we find

$$
\left\|\hat{\rho}_{1}\right\|^{\prime} \simeq 2 \rho_{0} \sqrt{\frac{\pi}{k_{0}}}\left(\frac{N}{\pi \rho}\right)^{3 / 4}\left|\cos \left(k_{0} R\right)\right| .
$$

As a result, we obtain

$$
\omega_{p}^{\prime}(\psi)=\frac{3}{4},
$$

which is different from the indices (29).

\subsection{One-dimensional case}

The condensate density matrix (21) takes the form

$$
\rho_{0}\left(x, x^{\prime}\right)=\rho_{0} \cos k_{0}\left(x-x^{\prime}\right),
$$

where $x \in(-\infty,+\infty)$. With the system length

$$
L \equiv \frac{N}{\rho}
$$


we have

$$
\left(\varphi_{k}, \hat{\rho}_{0} \varphi_{k}\right)=\rho_{0} L\left[\frac{\sin \left(\frac{k-k_{0}}{2} L\right)}{\left(k-k_{0}\right) L}+\frac{\sin \left(\frac{k+k_{0}}{2} L\right)}{\left(k+k_{0}\right) L}\right] .
$$

If $k_{0}=0$, then again we get $\omega_{p}(\psi)=1$, since

$$
\lim _{k_{0} \rightarrow 0}\left(\varphi_{k}, \hat{\rho}_{0} \varphi_{k}\right)=2 \frac{\rho_{0}}{k} \sin \left(\frac{k L}{2}\right)
$$

and the supremum over $k$ for the above right-hand side gives $N_{0}$.

The situation is rather interesting for $k_{0} \neq 0$. Then

$$
\sup _{k}\left(\varphi_{k}, \hat{\rho}_{0} \varphi_{k}\right)=\frac{\rho_{0} L}{2}\left[1+\frac{\sin \left(k_{0} L\right)}{k_{0} L}\right] .
$$

For a large system, with $k_{0} L \gg 1$, and, respectively, with a large number of particles, such that

$$
N \gg \frac{\rho}{k_{0}},
$$

we find

$$
\left\|\hat{\rho}_{1}\right\| \simeq \frac{\rho_{0}}{2 \rho} N
$$

Finally, we obtain

$$
\omega_{p}(\psi)=1 .
$$

When employing the trace norm (5), and using the equality

$$
\operatorname{Tr} \hat{\rho}_{0}^{2}=\frac{1}{2}\left(\rho_{0} L\right)^{2}\left(1+\frac{\sin k_{0} L}{k_{0} L}\right),
$$

we get for a large system

$$
\left\|\hat{\rho}_{1}\right\|^{\prime} \simeq \frac{\rho_{0}}{\sqrt{2} \rho} N
$$

This yields

$$
\omega_{p}^{\prime}(\psi)=1
$$

Hence, in the case of one-dimensional systems, the order indices (33), based on the spectral norm, coincide with the indices (34), based on the trace norm of reduced density matrices.

The order indices, defined through the spectral norm, or respectively through the trace norm, permit us to generalize the consideration to an arbitrary $d$-dimensional case resulting in

$$
\omega_{p}(\psi)=\frac{1}{d}, \quad \omega_{p}^{\prime}(\psi)=\frac{d+1}{2 d}
$$


This suggests a relation

$$
\omega_{p}^{\prime}(\psi)=\frac{1+\omega_{p}(\psi)}{2}
$$

between the order indices obtained for different norms.

Let us emphasize that the order indices provide us with a quantitative measure for the degree of order occurring in a statistical system. This measure makes it possible to unambiguously distinguish between the degrees of order of quite similar systems. For example, let us compare two three-dimensional systems, one with the condensate density matrix (23) and another with that matrix having the same algebraic decay, but without oscillations due to sine, that is when $\rho_{0}\left(\mathbf{r}, \mathbf{r}^{\prime}\right) \sim \rho_{0} / k_{0}\left|\mathbf{r}-\mathbf{r}^{\prime}\right|$. For the latter case, we find $\omega_{p}(\psi)=2 / 3$ instead of the index (25), which tells us that in the latter system the order is higher than in the system with the condensate matrix (23).

It is important that the order indices allow the description and classification of various types of mid-range order, as is illustrated in this section, when the order parameters are zero. Note that a Bose system with finite-momentum condensate [10-12], possessing mid-range order, satisfies the Cummings-Hyland-Rowlands relation [13].

\section{Condensation in confined systems}

The recent developments of Bose-Einstein condensation of trapped atoms (see reviews [14-16]) makes it important to consider how the order indices can be defined for finite systems. Stricktly speaking, in finite systems sharp phase transitions cannot occur. A rigorous definition of a genuine phase transition requires the usage of the thermodynamic limit (16). But the number of atoms in a trap, although large, however is always finite. Therefore the Bose-Einstein condensation in a trap is rather a gradual crossover than a sharp transition. The introduced matrix order indices do not necessarily require the employment of the thermodynamic limit, though their calculation is facilitated when the number of particles is large.

Bose-Einstein condensation can be treated as the appearance of a macroscopic fraction of atoms being in a coherent state. A coherent state, in general, is defined up to a phase factor with a random phase [17]. If this phase is made fixed, then the gauge symmetry in the system is broken. The breaking of gauge symmetry is usually done by means of the Bogolubov prescription [18] presenting the field operator as $\psi=\psi_{0}+\tilde{\psi}$, where $\psi_{0}$ is a classical quantity and $\tilde{\psi}$ is the field operator of noncondensed particles. In that case, the statistical average $\langle\psi\rangle=\psi_{0}$ is nonzero and might be considered as an order parameter. The gauge symmetry breaking implies that the number of particles in the system is not conserved. In reality, the number of particles in a system, even such as a trap, can be preserved with a rather good accuracy. Consequently, it would be logical to describe Bose-Einstein condensation without breaking gauge symmetry [19-24]. The suggested schemes, preserving particle conservation, are essentially more cumbersome then the techniques based on gauge symmetry breaking. This is why the latter techniques became more popular. However this way also contains intrinsic 
difficulties related with the appearance of divergences in low-order approximations. To compensate these divergences, one needs to invoke higher-order approximations [23], which again complicates the consideration. Here we advance a novel method of treating a system with Bose condensate, without breaking gauge symmetry. The method is sufficiently simple and general, and can be applied to trapped as well as untrapped atoms interacting through integrable potentials.

The pivotal idea of the method is the usage of random-phase coherent states [17]. When a fraction of atoms is in such a state, the field operator can be presented as the sum

$$
\psi(\mathbf{r}, t)=\eta_{\alpha}(\mathbf{r}, t)+\tilde{\psi}(\mathbf{r}, t)
$$

in which

$$
\eta_{\alpha}(\mathbf{r}, t)=\eta(\mathbf{r}, t) e^{i \alpha}
$$

is a random-phase coherent field [17] with $\alpha \in[0,2 \pi]$ being a random phase, and $\tilde{\psi}$ corresponds to incoherent particles.

The average of an operator $\hat{A}$ is defined as

$$
<\hat{A}>\equiv \frac{1}{2 \pi} \int_{0}^{2 \pi} \operatorname{Tr} \hat{\rho} \hat{A} d \alpha,
$$

including statistical averaging, with a statistical operator $\hat{\rho}$, and averaging over the random phases. From this definition, it follows that

$$
<\psi>=<\eta_{\alpha}>=<\tilde{\psi}>=0
$$

hence the gauge symmetry is not broken. The conservation of gauge symmetry is what makes the principal distinction between the presentation (36) and the Bogolubov prescription [18], where the gauge symmetry is broken. The coherent and incoherent terms in the sum (36) are subject to the normalization conditions

$$
\begin{gathered}
\int<\psi^{\dagger}(\mathbf{r}, t) \psi(\mathbf{r}, t)>d \mathbf{r}=N, \quad \int|\eta(\mathbf{r}, t)|^{2} d \mathbf{r}=N_{0}, \\
\int<\tilde{\psi}^{\dagger}(\mathbf{r}, t) \tilde{\psi}(\mathbf{r}, t)>d \mathbf{r}=\tilde{N},
\end{gathered}
$$

where $N_{0}$ is the number of coherent atoms, $\tilde{N}$ is the number of incoherent atoms, and the total number of atoms is

$$
N=N_{0}+\tilde{N} \text {. }
$$

The terms in the right-hand side of Eq. (36) are mutually orthogonal on the average, which means that

$$
<\eta_{\alpha}(\mathbf{r}, t) \tilde{\psi}\left(\mathbf{r}^{\prime}, t\right)>=0 .
$$

For the standard Hamiltonian with a two-particle interaction potential $\Phi(\mathbf{r})=$ $\Phi(-\mathbf{r})$, the Heisenberg equation for the field operator reads

$$
i \hbar \frac{\partial \psi}{\partial t}=H(\psi) \psi
$$


where $\psi=\psi(\mathbf{r}, t)$ and

$$
H(\psi)=-\frac{\hbar^{2} \nabla^{2}}{2 m_{0}}+U_{e x t}+U_{i n t}-\mu
$$

with $m_{0}$ being particle mass and $\mu$, chemical potential. The external potential $U_{\text {ext }}(\mathbf{r}, t)$ describes all external fields, including the confining potential and possible external perturbations. The interaction potential

$$
U_{\text {int }}(\mathbf{r}, t)=\int \Phi\left(\mathbf{r}-\mathbf{r}^{\prime}\right) \psi^{\dagger}\left(\mathbf{r}^{\prime}, t\right) \psi\left(\mathbf{r}^{\prime}, t\right) d \mathbf{r}^{\prime}
$$

is formed by particle interactions.

Let us substitute the presentation (36) into the Heisenberg equation (42). Then, let us either multiply Eq. (42) by $e^{-i \alpha}$ or just set $\alpha=0$, after which take the average $\langle\ldots\rangle$ according to definition (38). This results in the equation for the coherent field,

$$
\begin{gathered}
\left(i \hbar \frac{\partial}{\partial t}+\frac{\hbar^{2} \nabla^{2}}{2 m_{0}}-U_{e x t}+\mu\right) \eta(\mathbf{r}, t)= \\
=\int \Phi\left(\mathbf{r}-\mathbf{r}^{\prime}\right)\left[\left|\eta\left(\mathbf{r}^{\prime}, t\right)\right|^{2} \eta(\mathbf{r}, t)+\tilde{\rho}\left(\mathbf{r}^{\prime}, t\right) \eta(\mathbf{r}, t)+\tilde{\rho}_{1}\left(\mathbf{r}, \mathbf{r}^{\prime}, t\right) \eta\left(\mathbf{r}^{\prime}, t\right)\right] d \mathbf{r}^{\prime},
\end{gathered}
$$

in which the density of incoherent particles

$$
\tilde{\rho}(\mathbf{r}, t) \equiv \tilde{\rho}_{1}(\mathbf{r}, \mathbf{r}, t)
$$

is the diagonal element of the incoherent density matrix

$$
\tilde{\rho}_{1}\left(\mathbf{r}, \mathbf{r}^{\prime}, t\right) \equiv<\tilde{\psi}^{\dagger}\left(\mathbf{r}^{\prime}, t\right) \tilde{\psi}(\mathbf{r}, t)>
$$

Notice that in the equation (45) for the coherent field no anomalous averages arise, which is due to the gauge symmetry conservation.

If, after substituting the presentation (36) into the Heisenberg equation (42), we average only over the random phase, then we obtain the following equation

$$
\begin{gathered}
\left(i \hbar \frac{\partial}{\partial t}+\frac{\hbar^{2} \nabla^{2}}{2 m_{0}}-U_{e x t}+\mu\right) \tilde{\psi}(\mathbf{r}, t)= \\
=\int \Phi\left(\mathbf{r}-\mathbf{r}^{\prime}\right)\left[\tilde{\psi}^{\dagger}\left(\mathbf{r}^{\prime}, t\right) \tilde{\psi}\left(\mathbf{r}^{\prime}, t\right) \tilde{\psi}(\mathbf{r}, t)+\left|\eta\left(\mathbf{r}^{\prime}, t\right)\right|^{2} \tilde{\psi}(\mathbf{r}, t)+\eta^{*}\left(\mathbf{r}^{\prime}, t\right) \eta(\mathbf{r}, t) \tilde{\psi}\left(\mathbf{r}^{\prime}, t\right)\right] d \mathbf{r}^{\prime}
\end{gathered}
$$

for the incoherent term of the field operator. Let us stress that both equations (45) as well as (48) are exact.

One often obtains an equation for the condensate wave function, which is equivalent to Eq. (45), following what one calls the Popov approximation. In that way, one, first, breaks the gauge symmetry by means of the Bogolubov prescription and then one omits the anomalous averages arising because of the broken gauge symmetry. Such a way is 
clearly not self-consistent. Moreover, as is possible to show by direct calculations, in the case of broken gauge symmetry, anomalous averages are of the same order, or even larger, then normal averages [25,26]. Therefore, after the gauge symmetry has been broken, it is not correct to omit anomalous averages.

Given Eq. (48), we may derive an equation for the Green functions of incoherent particles. For this purpose, it is convenient to introduce the following short-hand notation [17]. Denote a function $f\left(\mathbf{r}_{1}, t_{1}\right)$ as $f(1)$ and the related differential measure as $d(1) \equiv d \mathbf{r}_{1} d t_{1}$. Then the Dirac delta-function is

$$
\delta(12) \equiv \delta\left(\mathbf{r}_{1}-\mathbf{r}_{2}\right) \delta\left(t_{1}-t_{2}\right) .
$$

Introduce the retarded interaction potential

$$
\Phi(12) \equiv \Phi\left(\mathbf{r}_{1}-\mathbf{r}_{2}\right) \delta\left(t_{1}-t_{2}+0\right)
$$

With this notation, the single-particle and two-particle causal Green functions are defined as

$$
G(12) \equiv-i<\hat{T} \tilde{\psi}(1) \tilde{\psi}^{\dagger}(2)>, \quad G(1234) \equiv-<\hat{T} \tilde{\psi}(1) \tilde{\psi}(2) \tilde{\psi}^{\dagger}(3) \tilde{\psi}^{\dagger}(4)>
$$

where $\hat{T}$ is the chronological operator. These are the Green functions at real times, which are directly related to the Matsubara Green functions at imaginary times $[17,27]$.

From definition (49) and Eq. (48), we immediately obtain the propagator equation

$$
\begin{gathered}
\left(i \hbar \frac{\partial}{\partial t_{1}}+\frac{\hbar^{2} \nabla_{1}^{2}}{2 m_{0}}-U_{e x t}+\mu\right) G(12)- \\
-\int \Phi(13)\left[|\eta(3)|^{2} G(12)+\eta^{*}(3) \eta(1) G(32)+i G_{2}(1332)\right] d(3)=\delta(12) .
\end{gathered}
$$

It is important to note that all Eqs. (45), (48), and (50) have sense only for the integrable interaction potentials, such that

$$
\left|\int_{V} \Phi(\mathbf{r}) d \mathbf{r}\right|<\infty
$$

In the other case, to avoid ultraviolet divergences, one has to set $\eta(\mathbf{r}, t) \equiv 0$, which implies the absence of condensate [17].

The propagator equation (50) can be written in a more compact form if one introduces the self-energy $\Sigma(12)$ by means of the relation

$$
\begin{gathered}
\int \Sigma(13) G(23) d(3)= \\
=\int \Phi(13)\left[|\eta(3)|^{2} G(12)+\eta^{*}(3) \eta(1) G(32)+i G_{2}(1332)\right] d(3) .
\end{gathered}
$$

From here, recalling the definition of the inverse propagator,

$$
\int G^{-1}(13) G(32) d(3)=\int G(13) G^{-1}(32) d(3)=\delta(12),
$$


we have the self-energy

$$
\begin{gathered}
\Sigma(12)=\delta(12) \int \Phi(13)|\eta(3)|^{2} d(3)+ \\
+\Phi(12) \eta(1) \eta^{*}(2)+i \int \Phi(13) G_{2}(1334) G^{-1}(42) d(34) .
\end{gathered}
$$

Then the inverse propagator is

$$
G^{-1}(12)=\left(i \hbar \frac{\partial}{\partial t_{1}}+\frac{\hbar^{2} \nabla_{1}^{2}}{2 m_{0}}-U_{e x t}+\mu\right) \delta(12)-\Sigma(12),
$$

and the propagator equation (50) takes the form

$$
\left(i \hbar \frac{\partial}{\partial t_{1}}+\frac{\hbar^{2} \nabla_{1}^{2}}{2 m_{0}}-U_{e x t}+\mu\right) G(12)-\int \Sigma(13) G(32) d(3)=\delta(12) .
$$

The pair of equations (45) and (55), together with the definition (53) for the incoherent self-energy, completely describes the Bose system with a coherent condensate. These equations, for a given interaction potential, are exact.

Dilute gases of trapped atoms are usually characterized by the Fermi contact potential

$$
\Phi(\mathbf{r})=A \delta(\mathbf{r}), \quad A \equiv 4 \pi \hbar^{2} \frac{a_{s}}{m_{0}},
$$

where $a_{s}$ is a scattering length.

In that case, the coherent-field equation (45) simplifies to

$$
i \hbar \frac{\partial \eta}{\partial t}=\left[-\frac{\hbar^{2} \nabla^{2}}{2 m_{0}}+U_{e x t}+A\left(|\eta|^{2}+2 \tilde{\rho}\right)-\mu\right] \eta,
$$

where $\eta=\eta(\mathbf{r}, t)$ and $\tilde{\rho}=\tilde{\rho}(\mathbf{r}, t)$. Equation (48) for the incoherent operator becomes

$$
i \hbar \frac{\partial \tilde{\psi}}{\partial t}=\left[-\frac{\hbar^{2} \nabla^{2}}{2 m_{0}}+U_{e x t}+A\left(\tilde{\psi}^{\dagger} \tilde{\psi}+2|\eta|^{2}\right)-\mu\right] \tilde{\psi}
$$

The self-energy (53) transforms to

$$
\Sigma(12)=A\left[2 \delta(12)|\eta(1)|^{2}+i \int G_{2}(1113) G^{-1}(32) d(3)\right],
$$

which enters the propagator equation (55).

To solve the propagator equation, one may follow one of the known iterative procedures $[17,28]$ starting e.g. with the Hartree-Fock approximation, when

$$
G_{2}(1234)=G(14) G(23)+G(13) G(24) .
$$

Then the self-energy (59) is

$$
\Sigma(12)=2 A \delta(12)\left[|\eta(1)|^{2}+\tilde{\rho}(1)\right] .
$$


If the external potential does not depend on time, $U_{e x t}=U(\mathbf{r})$, and the system is in equilibrium, then the coherent field may be presented as

$$
\eta(\mathbf{r}, t)=\sqrt{N_{0}} \varphi(\mathbf{r}) \exp \left(-\frac{i}{\hbar} E t\right),
$$

with the function $\varphi(\mathbf{r})$ normalized to unity, $(\varphi, \varphi)=1$. In this case, Eq. (57) reduces to the stationary equation

$$
\hat{H}(\varphi) \varphi(\mathbf{r})=E \varphi(\mathbf{r}),
$$

with the effective Hamiltonian

$$
\hat{H}(\varphi) \equiv-\frac{\hbar^{2} \nabla^{2}}{2 m_{0}}+U+A\left(N_{0}|\varphi|^{2}+2 \tilde{\rho}\right)-\mu .
$$

Note that this Hamiltonian depends on thermodynamic parameters, such as temperature, through the incoherent density $\tilde{\rho}=\tilde{\rho}(\mathbf{r})$.

In the presence of a confining potential, the spectrum of the eigenproblem (62) is discrete [29]. For a system in equilibrium, the condensate energy $E$ corresponds to the lowest energy level of the eigenproblem (62). For a nonequilibrium system, one can excite higher nonlinear coherent modes corresponding to higher states of Eq. (62), thus, creating nonground-state coherent condensates [29-33].

For an equilibrium system, the single-particle density matrix

$$
\rho_{1}\left(\mathbf{r}, \mathbf{r}^{\prime}\right)=\left\langle\psi^{\dagger}\left(\mathbf{r}^{\prime}\right) \psi(\mathbf{r})>\right.
$$

is the sum

$$
\rho_{1}\left(\mathbf{r}, \mathbf{r}^{\prime}\right)=\rho_{0}\left(\mathbf{r}, \mathbf{r}^{\prime}\right)+\tilde{\rho}_{1}\left(\mathbf{r}, \mathbf{r}^{\prime}\right)
$$

of the coherent term

$$
\rho_{0}\left(\mathbf{r}, \mathbf{r}^{\prime}\right) \equiv N_{0} \varphi_{0}(\mathbf{r}) \varphi_{0}^{*}\left(\mathbf{r}^{\prime}\right)
$$

where $\varphi_{0}(\mathbf{r})$ is the ground state of the eigenproblem (62), and of the incoherent density matrix (47). The coherent density matrix $\hat{\rho}_{0}=\left[\rho_{0}\left(\mathbf{r}, \mathbf{r}^{\prime}\right)\right]$ has the properties

$$
\hat{\rho}^{p}=N_{0}^{p-1} \hat{\rho}_{0}, \quad \operatorname{Tr} \hat{\rho}_{0}^{p}=N_{0}^{p} .
$$

The incoherent density matrix can be presented as an expansion

$$
\tilde{\rho}_{1}\left(\mathbf{r}, \mathbf{r}^{\prime}\right)=\sum_{k} n_{k} \varphi_{k}(\mathbf{r}) \varphi_{k}^{*}\left(\mathbf{r}^{\prime}\right)
$$

over natural wave functions [3] corresponding to incoherent states.

When the coherent condensate is absent, $N_{0} \equiv 0$, then the spectral norm of $\hat{\rho}_{1}$ is $\left\|\hat{\rho}_{1}\right\|=\sup _{k} n_{k}$, which is finite. The trace norm gives $\left\|\hat{\rho}_{1}\right\|^{\prime}=\sqrt{\sum_{k} n_{k}^{2}}$, which is of order $N^{1 / 2}$. The related order indices are $\omega_{p}(\psi)=0$ and $\omega_{p}^{\prime}(\psi)=1 / 2$, respectively. However, as soon as $N_{0}>\sup _{k} n_{k}$, then for a large system with $N \gg 1$, one has

$$
\omega_{p}(\psi)=\omega_{p}^{\prime}(\psi)=\frac{\ln N_{0}}{\ln N} .
$$


In the thermodynamic limit (16), we return to the order indices (18). But for a finite system the order indices (65) are, in general, functions of thermodynamic variables, such as temperature and density. When $N_{0}$ changes from 1 to $N$, the order indices increase from 0 to 1 , which corresponds to a gradual crossover.

Recall that the present consideration has been based on the decomposition (36) for the field operator onto its coherent and incoherent parts. This decomposition is valid only when the interaction potential is integrable, satisfying condition (51). The Fermi contact potential (56) is integrable. This potential describes pair interactions in dilute gases, whose mean interatomic distance is much larger than the magnitude of the scattering length $\left|a_{s}\right|$. For dense gases and liquids, interaction potentials often contain hard cores and are not integrable. In such cases, the decomposition (36) is not appropriate and one has to invoke other calculational techniques explicitly taking account of interatomic correlations (see e.g. [34-36] and references in review [16]).

\section{Magnetic phase transitions}

The matrix indices (17), defined for reduced density matrices, are convenient for characterizing such phase transitions as Bose condensation and superconductivity. The question that one could pose is whether the same indices (17) would be suitable for describing magnetic phase transitions. And also, since superconductivity and magnetic order can coexist $[37,39]$, could the indices (17) characterize such a coexistence of orders? In this section, we show that the order indices (17), based on reduced density matrices (18), are not suitable for describing magnetic order. To describe the latter, it is necessary to return to the general definition (1) of the matrix order indices and to introduce the matrices composed of spin operators.

Consider, first, the indices $\omega_{p}(\psi)$. Let the field operator be a spinor $\psi=\left[\psi_{\sigma}\right]$, with $\sigma=\uparrow, \downarrow$ denoting spin up or down. Keeping in mind a solid with a crystalline lattice, we enumerate the lattice sites by the index $i=1,2, \ldots, N$. Wave functions, localized at the related sites, are called the localized orbitals, such as Wannier functions. For simplicity, we shall deal with the single-zone case. Let $\left\{\varphi_{i}(\mathbf{r})\right\}$ be a set of localized orbitals that are orthonormalized, $\left(\varphi_{i}, \varphi_{j}\right)=\delta_{i j}$, and asymptotically complete, which means that

$$
\sum_{i=1}^{N} \varphi_{i}(\mathbf{r}) \varphi_{i}^{*}\left(\mathbf{r}^{\prime}\right)=\delta_{N}\left(\mathbf{r}-\mathbf{r}^{\prime}\right),
$$

with the right-hand side asymptotically, as $N \rightarrow \infty$, coinciding with the $\delta$-function,

$$
\lim _{N \rightarrow \infty} \delta_{N}(\mathbf{r})=\delta(\mathbf{r})
$$

The field operator $\psi_{\sigma}(\mathbf{r})$ can be expanded over the localized orbitals as

$$
\psi_{\sigma}(\mathbf{r})=\sum_{i=1}^{N} c_{i \sigma} \varphi_{i}(\mathbf{r})
$$


Both operators $\psi_{\sigma}(\mathbf{r})$ as well as $c_{i \sigma}$ satisfy the Fermi commutation relations. To stress that the particles are well localized, the following localization conditions are imposed on the operators $c_{i \sigma}$ :

$$
c_{i \sigma}^{\dagger} c_{j \sigma^{\prime}}=\delta_{i j} c_{i \sigma}^{\dagger} c_{i \sigma^{\prime}}, \quad c_{i \sigma}^{\dagger} c_{j \sigma^{\prime}}^{\dagger} c_{f \sigma^{\prime}} c_{g \sigma}=\delta_{i g} \delta_{j f} c_{i \sigma}^{\dagger} c_{j \sigma^{\prime}}^{\dagger} c_{j \sigma^{\prime}} c_{i \sigma}+\delta_{i f} \delta_{j g} c_{i \sigma}^{\dagger} c_{j \sigma^{\prime}}^{\dagger} c_{i \sigma^{\prime}} c_{j \sigma} .
$$

Limiting the number of particles at each site by one, we have the unipolarity conditions

$$
\sum_{\sigma} c_{i \sigma}^{\dagger} c_{i \sigma}=1, \quad c_{i \sigma} c_{i \sigma^{\prime}}=0
$$

From the particle operators $c_{i \sigma}$, one can pass to spin operators $S_{i}^{\alpha}$ by means of the transformations

$$
\begin{aligned}
c_{i \uparrow}^{\dagger} c_{i \uparrow}=\frac{1}{2}+S_{i}^{z}, & c_{i \downarrow}^{\dagger} c_{i \downarrow}=\frac{1}{2}-S_{i}^{z}, \\
c_{i \uparrow}^{\dagger} c_{i \downarrow}=S_{i}^{+} \equiv S_{i}^{x}+i S_{i}^{y}, & c_{i \downarrow}^{\dagger} c_{i \uparrow}=S_{i}^{-} \equiv S_{i}^{x}-i S_{i}^{y} .
\end{aligned}
$$

Now, we construct the reduced density matrices (13). The single-particle density matrix is

$$
\rho_{1}\left(\mathbf{r}, \mathbf{r}^{\prime}\right)=\delta_{N}\left(\mathbf{r}-\mathbf{r}^{\prime}\right),
$$

where $\delta_{N}(\mathbf{r})$ is the asymptotic $\delta$-function, appearing in Eq. (66). Note that, because of $\operatorname{Tr} \hat{\rho}_{1}=N$, one has $\delta_{N}(0) \equiv N / V$. The spectral and trace norms, respectively, give

$$
\left\|\hat{\rho}_{1}\right\| \simeq 1, \quad\left\|\hat{\rho}_{1}\right\|^{\prime} \simeq \sqrt{N} \quad(N \rightarrow \infty)
$$

From here

$$
\omega_{1}(\psi)=0, \quad \omega_{1}^{\prime}(\psi)=\frac{1}{2},
$$

independently from the existence or absence of magnetic order.

For the two-particle reduced density matrix, we have

$$
\begin{gathered}
\rho_{2}\left(\mathbf{r}_{1}, \mathbf{r}_{2}, \mathbf{r}_{1}^{\prime}, \mathbf{r}_{2}^{\prime}\right)=\rho_{1}\left(\mathbf{r}_{1}, \mathbf{r}_{1}^{\prime}\right) \rho_{1}\left(\mathbf{r}_{2}, \mathbf{r}_{2}^{\prime}\right)-\frac{1}{2} \rho_{1}\left(\mathbf{r}_{1}, \mathbf{r}_{2}^{\prime}\right) \rho_{1}\left(\mathbf{r}_{2}, \mathbf{r}_{1}^{\prime}\right)- \\
-2 \sum_{i \neq j}^{N}<\mathbf{S}_{i} \cdot \mathbf{S}_{j}>\varphi_{i}\left(\mathbf{r}_{1}\right) \varphi_{j}\left(\mathbf{r}_{2}\right) \varphi_{j}^{*}\left(\mathbf{r}_{1}^{\prime}\right) \varphi_{i}^{*}\left(\mathbf{r}_{2}^{\prime}\right) .
\end{gathered}
$$

To simplify the consideration, let the particle interactions be of long-range type, for which the asymptotic, as $N \rightarrow \infty$, decoupling

$$
<\mathbf{S}_{i} \cdot \mathbf{S}_{j}>\approx \mathbf{S}_{i}><\mathbf{S}_{j}>\quad(i \neq j)
$$

is valid. And also let the lattice be ideal, when, because of the translation invariance, one has

$$
<\mathbf{S}_{i}>=<\mathbf{S}>, \quad \mathbf{S} \equiv \frac{1}{N} \sum_{i=1}^{N} \mathbf{S}_{i}
$$


Then the two-particle matrix (73) becomes

$$
\rho_{2}\left(\mathbf{r}_{1}, \mathbf{r}_{2}, \mathbf{r}_{1}^{\prime}, \mathbf{r}_{2}^{\prime}\right)=\rho_{1}\left(\mathbf{r}_{1}, \mathbf{r}_{1}^{\prime}\right) \rho_{1}\left(\mathbf{r}_{2}, \mathbf{r}_{2}^{\prime}\right)-\left(\frac{1}{2}+2<\mathbf{S}>^{2}\right) \rho_{1}\left(\mathbf{r}_{1}, \mathbf{r}_{2}^{\prime}\right) \rho_{1}\left(\mathbf{r}_{2}, \mathbf{r}_{1}^{\prime}\right)
$$

The eigenfunctions of this matrix are

$$
\varphi_{i j}\left(\mathbf{r}, \mathbf{r}^{\prime}\right)=\frac{1}{\sqrt{2}}\left[\varphi_{i}(\mathbf{r}) \varphi_{j}\left(\mathbf{r}^{\prime}\right)-\varphi_{i}\left(\mathbf{r}^{\prime}\right) \varphi_{j}(\mathbf{r})\right]
$$

with $\varphi_{i}(\mathbf{r})$ being the localized orbitals. For a large system, we get

$$
\left\|\hat{\rho}_{2}\right\| \simeq \frac{3}{2}+2<\mathbf{S}>^{2}, \quad\left\|\hat{\rho}_{2}\right\|^{\prime} \sim N
$$

as $N \rightarrow \infty$. Thus, in the thermodynamic limit, we find

$$
\omega_{p}(\psi)=0, \quad \omega_{p}^{\prime}(\psi)=\frac{1}{2}
$$

This shows that, although the norms of the reduced density matrices do change under the arising magnetic order, that change is negligible in the thermodynamic limit. Hence, the indices $\omega_{p}(\psi)$ are not suitable for describing magnetic phase transitions.

This problem can easily be resolved by going back to the more general definition of the matrix indices (10). Being interested in magnetic order, it is reasonable to work with correlation functions composed of spin operators. For instance, the two-spin correlator can be written as

$$
D_{i j}(S) \equiv<\mathbf{S}_{j} \cdot \mathbf{S}_{i}>
$$

In general, the $2 p$-spin correlator is

$$
D_{i_{1} \ldots i_{p} j_{1} \ldots j_{p}}(S) \equiv<\mathbf{S}_{j_{p}} \cdots\left(\mathbf{S}_{j_{1}} \cdot \mathbf{S}_{i_{1}}\right) \cdots \mathbf{S}_{i_{p}}>
$$

The related spin-correlation matrices $\hat{D}_{p}(S)$ are the matrices with the elements $(77)$. For example, $\hat{D}_{1}(S)=\left[D_{i j}(S)\right]$ is an $N \times N$ matrix with the elements (76).

For a system defined on an ideal lattice, the natural wave functions are

$$
\varphi_{k}\left(\mathbf{a}_{i}\right)=\frac{1}{\sqrt{N}} e^{i \mathbf{k} \cdot \mathbf{a}_{i}}
$$

with $\mathbf{a}_{i}$ being a lattice vector. These functions form an orthonormalized basis, for which

$$
\sum_{i} \varphi_{k}^{*}\left(\mathbf{a}_{i}\right) \varphi_{k^{\prime}}\left(\mathbf{a}_{i}\right)=\delta_{k k^{\prime}}, \quad \sum_{k} \varphi_{k}\left(\mathbf{a}_{i}\right) \varphi_{k}^{*}\left(\mathbf{a}_{j}\right)=\delta_{i j}
$$

Calculating the spectral norm of $\hat{D}_{1}(S)$, we find

$$
\left\|\hat{D}_{1}(S)\right\|=\sup _{k}\left[S(S+1)+\sum_{j=1}^{N-1}<\mathbf{S}_{j} \cdot \mathbf{S}_{i}>e^{i \mathbf{k} \cdot \mathbf{a}_{j}}\right] .
$$


The trace norm yields

$$
\left\|\hat{D}_{1}(S)\right\|^{\prime}=\left(N \sum_{j=1}^{N-1}<\mathbf{S}_{j} \cdot \mathbf{S}_{0}>\right)^{1 / 2}
$$

And for the trace, we have

$$
\operatorname{Tr} \hat{D}_{1}(S)=S(S+1) N
$$

When magnetic order is absent, i.e. $\langle\mathbf{S}>=0$, then for a large system, with $N \gg 1$,

$$
\left\|\hat{D}_{1}(S)\right\| \sim 1, \quad\left\|\hat{D}_{1}(S)\right\|^{\prime} \sim N^{1 / 2}
$$

Hence, generalizing this, we get

$$
\omega_{p}(S)=0, \quad \omega_{p}^{\prime}(S)=\frac{1}{2} \quad(<\mathbf{S}>=0) .
$$

But as soon as there appear magnetic order, then

$$
\left\|\hat{D}_{1}(S)\right\| \simeq N<\mathbf{S}>^{2}, \quad\left\|\hat{D}_{1}(S)\right\|^{\prime} \simeq N|<\mathbf{S}>|
$$

as $N \rightarrow \infty$. In this way, we come to the order indices

$$
\omega_{p}(S)=\omega_{p}^{\prime}(S)=1 \quad(<\mathbf{S}>\neq 0) .
$$

Comparing Eqs. (79) and (80), we see that the order indices $\omega_{p}(S)$ perfectly distinguish between the existence and absence of magnetic order. The description of the latter by the indices $\omega_{p}(S)$ is analogous to the characterization of Bose condensation by the indices $\omega_{p}(\psi)$. This is not surprising, since the arising magnetic order can be interpreted as condensation of magnons [39].

\section{Transitions with diagonal order}

The eigenvalues of the reduced density matrices $\hat{\rho}_{p}$ are known to be sensitive to the existence of the off-diagonal long-range order [3-6]. This is why the indices $\omega_{p}(\psi)$ are so useful for characterizing phase transitions with arising off-diagonal order, such as superconductivity and Bose condensation. Similarly, the indices $\omega_{p}(S)$ are convenient for describing magnetic transitions. These indices, however, are not sensitive to the appearance of the so-called diagonal long-range order, associated with the properties of the diagonal elements of density matrices. Among phase transitions with developing diagonal order, we may mention the melting-crystallization phase transition, which is so well known on earth and, presumably, occurring in neutron stars [40]. The mixingstratification transformation is also of this kind.

The difference between phase transitions with off-diagonal long-range order and diagonal long-range order is in the following. In the former case, the largest eigenvalue 
of one of the reduced density matrices, and respectively the related spectral norm, change from being of order one to the order of $N^{p}$. Such a dramatic variation of the norm, due to the arising off-diagonal order, immediately results in the sharp change of the order index $\omega_{p}(\psi)$. But so drastic increase of eigenvalues of the reduced density matrices does not happen under the appearing diagonal order, when all eigenvalues of $\hat{\rho}_{p}$ remain of order one. Thus, for both crystalline and liquid states

$$
\left\|\hat{\rho}_{p}\right\| \sim 1, \quad\left\|\hat{\rho}_{p}\right\|^{\prime} \sim N^{1 / 2} .
$$

Therefore the indices

$$
\omega_{p}(\psi)=0, \quad \omega_{p}^{\prime}(\psi)=\frac{1}{2}
$$

do not notice that the diagonal order has appeared.

Fortunately, with the general definition of the matrix order indices (1) or (10), we are not obliged to deal exclusively with the density matrices (12). It is always possible to define such a matrix whose order index would be sensitive to any kind of arising order.

As an illustration, let us consider the crystallization transition. Introduce the density-difference operator

$$
\Delta(\mathbf{r}) \equiv \psi^{\dagger}(\mathbf{r}) \psi(\mathbf{r})-\rho,
$$

with $\rho \equiv N / V$ being the average density. Statistical averaging of the operator (82) gives

$$
<\Delta(\mathbf{r})>=\rho(\mathbf{r})-\rho, \quad \rho(\mathbf{r}) \equiv<\psi^{\dagger}(\mathbf{r}) \psi(\mathbf{r})>.
$$

Define the matrix $\hat{D}_{1}(\Delta)$ whose elements are

$$
D_{1}\left(\Delta, \mathbf{r}, \mathbf{r}^{\prime}\right)=<\Delta\left(\mathbf{r}^{\prime}\right) \Delta(\mathbf{r})>\text {. }
$$

Similarly, we may construct a matrix $\hat{D}_{p}(\Delta)$ of any order $p=1,2, \ldots$, as is explained in Eq. (6). The elements of $\hat{D}_{p}(\Delta)$ satisfy the correlation weakening condition, e.g.

$$
<\Delta(\mathbf{r}) \Delta\left(\mathbf{r}^{\prime}\right)>\simeq<\Delta(\mathbf{r})><\Delta\left(\mathbf{r}^{\prime}\right)>\quad\left(\left|\mathbf{r}-\mathbf{r}^{\prime}\right| \rightarrow \infty\right)
$$

In the case of liquid, with uniform density, we have

$$
<\Delta(\mathbf{r})>=0, \quad \rho(\mathbf{r})=\rho .
$$

Eigenvalues of $\hat{D}_{1}(\Delta)$ are of order 1 , which yields

$$
\left\|\hat{D}_{1}(\Delta)\right\| \sim 1, \quad\left\|\hat{D}_{1}(\Delta)\right\|^{\prime} \sim N^{1 / 2} .
$$

Following this way, we find

$$
\omega_{p}(\Delta)=0, \quad \omega_{p}^{\prime}(\Delta)=\frac{1}{2},
$$

which signifies the absence of order. 
The occurrence of crystalline order is marked by a well defined crystalline lattice spanned by the lattice vectors $\mathbf{a}_{i}$, with $i=1,2, \ldots, N$. Introduce the function $D_{1}\left(\Delta, \mathbf{a}_{i}, \mathbf{a}_{j}\right)$ as in Eq. (83) and the related matrix $\hat{D}_{1}(\Delta)=\left[D_{1}\left(\Delta, \mathbf{a}_{i}, \mathbf{a}_{j}\right)\right]$, which is an $N \times N$ matrix. The natural wave functions for a lattice system are presented in Eq. (78). Employing the spectral norm, we get

$$
\left\|\hat{D}_{1}(\Delta)\right\| \simeq N[\rho(\mathbf{a})-\rho]^{2} \quad(N \rightarrow \infty),
$$

where $\mathbf{a}$ is any of $\mathbf{a}_{i}$, since for an ideal lattice, $\rho\left(\mathbf{a}_{i}\right)=\rho\left(\mathbf{a}_{j}\right)$. The same expression follows for the trace norm $\left\|\hat{D}_{1}(\Delta)\right\|^{\prime}$. Finally, we obtain

$$
\omega_{p}(\Delta)=\omega_{p}^{\prime}(\Delta)=1
$$

As is seen, the formation of crystalline order leads to the change of the matrix indices $\omega_{p}(\Delta)$ from the values in Eq. (84) to those in Eq. (85).

As s brief conclusion, let us stress that the matrix order indices, introduced in the present paper, is a new general notion allowing for the description and classification of arbitrary types of order, off-diagonal as well as diagonal, long-range and mid-range, occurring in infinite as well as finite systems, and happening in different spaces (real, momentum, spin,...). The matrix order indices can be well defined when the order parameters do not exist. These indices are suitable for describing sharp phase transitions as well as gradual crossovers.

\section{Acknowledgement}

I am grateful to A.J. Coleman for numerous useful discussions. A Grant of the São Paulo State Research Foundation, Brazil, is appreciated. 


\section{References}

[1] D. ter Haar, Rep. Prog. Phys. 24 (1961) 304.

[2] K. Blum, Density Matrix Theory and Applications, Plenum, New York, 1981.

[3] A.J. Coleman, V.I. Yukalov, Reduced Density Matrices, Springer, Berlin, 2000.

[4] O. Penrose, Philos. Mag. 42 (1951) 1373.

[5] O. Penrose, L. Onsager, Phys. Rev. 104 (1956) 576.

[6] C.N. Yang, Rev. Mod. Phys. 34 (1962) 694.

[7] A. J. Coleman, V.I. Yukalov, Mod. Phys. Lett. B 5 (1991) 1679.

[8] A.J. Coleman, V.I. Yukalov, Nuovo Cimento B 108 (1993) 1377.

[9] A.J. Coleman, V.I. Yukalov, Int. J. Mod. Phys. B 10 (1996) 3505.

[10] V.I. Yukalov, Theor. Math. Phys. 37 (1978) 1093.

[11] V.I. Yukalov, Physica A 100 (1980) 431.

[12] V.I. Yukalov, Phys. Lett. A 83 (1981) 26.

[13] F.W. Cummings, C.J. Hyland, G. Rowlands, Phys. Lett. A 86 (1981) 370.

[14] A.S. Parkins, D.F. Walls, Phys. Rep. 303 (1998) 1.

[15] F. Dalfovo, S. Giorgini, L.P. Pitaevskii, S. Stringari, Rev. Mod. Phys. 71 (1999) 463.

[16] P.W. Courteille, V.S. Bagnato, V.I. Yukalov, Laser Phys. 11 (2001) 659.

[17] V.I. Yukalov, Statistical Green's Functions, Queen's University, Kingston, 1998.

[18] N.N. Bogolubov, Lectures on Quantum Statistics, Vol. 1, Gordon and Breach, New York, 1967.

[19] M. Girardeau and R. Arnowitt, Phys. Rev. 113 (1959) 755.

[20] C.W. Gardiner, Phys. Rev. A 56 (1997) 1414.

[21] Y. Castin, R. Dum, Phys. Rev. A57 (1998) 3008.

[22] M. Girardeau, Phys. Rev. A 58 (1998) 775.

[23] S.A. Morgan, J. Phys. B 33 (2000) 3847.

[24] A.E. Ruckenstein, Found. Phys. 30 (2000) 2113. 
[25] A. Griffin, Phys. Rev. B 22 (1980) 5193.

[26] T. Isoshima, K. Machida, J. Phys. Soc. Jap. 66 (1997) 3502.

[27] G. Cuniberti, E. De Micheli, G.A. Viano, Commun. Math. Phys. 216 (2001) 59.

[28] R. Del Sole, L. Reining, R.W. Godby, Phys. Rev. B 49 (1994) 8024.

[29] V.I. Yukalov, E.P. Yukalova, V.S. Bagnato, Phys. Rev. A 56 (1997) 4845.

[30] V.I. Yukalov, E.P. Yukalova, V.S. Bagnato, Laser Phys. 10 (2000) 26.

[31] V.S. Bagnato, E.P. Yukalova, V.I. Yukalov in: Bose-Einstein Condensates and Atom Lasers, eds. S. Martellucci, A. Chester, A. Aspect, M. Inguscio, Kluwer, New York, 2000, p. 201.

[32] V.I. Yukalov, E.P. Yukalova, V.S. Bagnato, Laser Phys. 11 (2001) 455.

[33] V.I. Yukalov, E.P. Yukalova, V.S. Bagnato, Proc. Int. Soc. Opt. Eng. 4243 (2001) 150 .

[34] E. Manousakis, V.K. Pandharipande, Q.N. Usmani, Phys. Rev. B 31 (1985) 7022.

[35] C. Carraro, S.E. Koonin, Phys. Rev. Lett. 65 (1990) 2792.

[36] E. Manousakis, V.K. Pandharipande, Q.N. Usmani, Phys. Rev. B 43 (1991) 13587.

[37] I. Felner, U. Asaf, Y. Levi, O. Millo, Phys. Rev. B 55 (1997) 3374.

[38] I. Felner, U. Asaf, C. Godart, E. Alleno, Physica B 259 (1999) 703.

[39] V.I. Yukalov, A.S. Shumovsky, Lectures on Phase Transitions, World Scientific, Singapore, 1990.

[40] N.K. Glendenning, Phys. Rep. 342 (2001) 393. 